\title{
Pre-Published Copy
}

Reference: Thomas, G.M. (2012) Rethinking Disability: Bodies, Senses, and Things, by Michael Schillmeier. Reviewed in: The Sociological Review 60(3), pp. 575-577.

In Rethinking Disability, Michael Schillmeier offers a comprehensive analysis of how bodies, senses and things connect to everyday practices and configure both 'enabling and disabling' (p. 15) circumstances. Focusing almost exclusively on blind individuals and conducting his analysis through an Science and Technology Studies lens, Schillmeier contributes to his existing work which explores ideas surrounding bodies and dis/ability (Schillmeier 2009), cosmo-political events (Schillmeier and Pohler, 2006), and the societal relevance of objects (Schillmeier 2007). The book itself underscores the historical breakthrough of understanding disablement as a social reality, highlighting the limitations of the social model of disability embedded within our current visual culture. Although supporting the counter-politics of the social model of disability, Schillmeier openly denounces its current structure as monolithic and as mistakenly presuming that the 'social' (p. 2) is a self-contained unit which can universally solve every quandary of the disability question. Despite Schillmeier often being guilty of labouring this point, his contention that the social necessitates a more complex explanation which accounts for the practices and experiences of disablement is calculated, revealing, and undoubtedly champions the use of actor-network theory in comprehending everyday practices.

The book is organised into three parts, eight chapters in all. The introduction succinctly devotes itself to tracing the trajectories of the social model of disability, a model which identifies systematic barriers, prejudice, and social exclusion imposed by society and its members. Schillmeier, extending his argument into Part I of the book, draws on pertinent and prominent literature to outline the primary tenets of the social model of disability and its focus on the material production of disability by certain organisational structures. He convincingly denounces a model which endorses exclusivist dualisms (disability and impairment, the natural and the social) and can be read as a counter-productive either/or strategy in comprehending the multifaceted and highly-situated experience of disability. This, Schillmeier contends, brings about the 
artificial fixation of disability into two spheres of reality, namely, the social and nonsocial reality of disablement. Schillmeier elaborates on this theme with an instructive exploration of how our divisions and modern blindness interact with the light culture as invoked by the ancient Greeks. It is to his credit that Schillmeier fruitfully engages with an appreciably difficult subject matter, albeit not always transparently, and persuasively explicates how a modern understanding of blindness abstracts and excludes the complexities of blind people's life by promoting, and preserving, the division of the social and non-social.

Schillmeier, in calling for an understanding of disability - and particularly blindness which moves towards a consideration of the 'religiousness' (p. 9) of relations, reaffirms how disability is a practice beyond both privation and the result of oppressive societal structures. Drawing on philosophical and sociological literature, he convincingly advances that the disability field, seeing beyond these bifurcated limits, would benefit by turning to the multiplicity of everyday practices. The discussion of Heidegger and Nietzsche, in an attempt to bring back a philosophical ethos of the empirical, could be trying for those unfamiliar with these works. Fortunately, Schillmeier's manifesto for a new understanding of disability in Part II of the book is less demanding, perhaps even more rewarding. The manifesto advances that the social needs to be explained by the very everyday practices which render people, purposefully and inadvertently, as enabled or disabled. This argument could again be justly subjected to accusations of protraction, though its significance should not be discounted. By formulating a conceptual understanding of the social of disability with reference to blindness, Schillmeier successfully highlights the limits of the social model of disability, an abstract and idealistic methodological framing of reality which wrongly reduces the complexity of reality by disregarding other prominent domains such as the medical perspective. This sophisticated rallying cry draws attention to 'how bodies, senses, and things connect or disconnect, enable or disable, provoke good as well as bad experiences' (p. 118).

Part III of the book supports Schillmeier's demands for an alternative understanding of disability by tracing the empirical complexities of blindness. This is the most 
enlightening and resourceful portion of the book in which Schillmeier recounts the findings obtained during a qualitative study of blind individuals and the use of technologies in their everyday lives. His concluding chapters begin with an examination of the disabling spaces of calculation, thereby drawing attention to how 'ordinary acts of everyday life make up the complex and contingent scenarios of dis/abilities that create enabling and disabling practices' (p. 127). To specify his argument, Schillmeier illustrates the way by which money and money technologies (ATMs, coins, cards) make up highly disabling practices in the life of blind people. The interviews clearly expound how the readability of money inscriptions become problematic, with the ordinary medium of exchange generating disabling scenarios which render individuals as disabled. This is endorsed with interesting empirical examples of how blind people operate within risky spaces of money at night-time. One of the most accessible passages establishes the importance of blind persons transforming 'bad money' into 'good money' (p. 142), containing revealing anecdotes of the ways in which blind persons choose notes over coins, establish separate pockets for separate coins, rely on humans and nonhuman technologies, and manage trust during transactions. Heidegger's construct of time-space is used to compliment an explanation of shopping and independency, yet the reader may find the analysis of Goffman more accessible. Schillmeier captures how blind individuals disrupt and make visible taken-for-granted practices, a perspective which clearly shares an affiliation with the principles of ethnomethodology (Garfinkel 1967) yet, interestingly, one which Schillmeier does not explicitly recognise.

The empirical examples cited successfully elucidate how the mediation of heterogeneous times and spaces, of dependencies on others which may be human and nonhuman alike (partners, the white cane), create enabling and disabling scenarios for the blind. What comes into view is how and in what moments disability is an effect of what Latimer (2009) calls body-world relations. This analysis is extended into Schillmeier's final chapter in which he again explicates the futility of complying with distinctive and hermetic dualisms such as dependence or independence, impairment or disability, social or nature, which divides disability into an individual or socio-cultural attributed entity and thus cannot make the world present. This approach, so avidly 
championed by Schillmeier, draws attention to the "crucial importance of the relations between human feeling, sensory and sensuous bodies, and nonhuman technologies, artefacts, and objects' (p. 171) which make up disabling practices. The use of a (symbolic) white cane alongside other technologies, together with the idea that blind persons are produced and socialised to elude the assistance of humans and nonhumans, is fascinating enough to merit an exploration which goes beyond the brevity it is currently afforded.

Although the book would be improved with further empirical examples accompanied by greater care taken in not exhausting some of the primary arguments, Schillmeier's skill is in producing an animated and informative analysis which represents a credible retort to frequently insular and rigid studies of disablement, together with providing an extensive argument in favour of developing an actor-network approach. The reader is presented with a coherent argument early in the text, followed by a convincing blend of research and relevant literature which gradually validate the main contentions. The book is recommended for disability advocates and postgraduate students engaging with disability studies for the first time, and will undoubtedly prove a useful tool in the fields of Science and Technology Studies, medical sociology, and the sociology of the body. Whilst Schillmeier makes no secret of being an apologist for disabled persons, his affiliation has little impact on producing a balanced and well thought-out argument. Removed yet engaging, dense yet accessible, the book is a welcome addition to the field. As bawled early in the book, 'disability matters!' (p. $11)$.

Gareth Thomas

Cardiff University

\section{References}

Garfinkel, H. (1967), Studies in Ethnomethodology, New Jersey: Prentice Hall. 
Latimer, J., (2009), 'Introduction: Body, Knowledge, World', in Latimer, J. and Schillmeier, M. (eds), Un/Knowing Bodies, Oxford: Blackwell: pp. 1-22.

Schillmeier, M., (2007), 'Dis/abling Spaces of Calculation: Blindness and Money in Everyday Life', Environment and Planning D: Society \& Space, 25 (4): 594-609.

Schillmeier, M., (2009), 'Actor Networks of Dementia', in Latimer, J. and Schillmeier, M. (eds), Un/Knowing Bodies, Oxford: Blackwell: pp. 141-158.

Schillmeier, M. and Pohler, W., (2006), 'Kosmo-Politische Ereignisse. Zur Sozialen Topologie von SARS', Soziale Welt, 57 (4): 331-349. 